\title{
Trisomy 22. Two new cases and delineation of the phenotype*
}

\author{
VICTOR B. PENCHASZADEH and ROBERTO COCO \\ Sección de Genética Médica and Centro de Investigaciones Endocrinológicas, Policlinico de Niños, Buenos Aires, \\ Argentina
}

\begin{abstract}
Summary. Two unrelated children, not affected with Down's syndrome, with strikingly similar phenotypes and an extra G-like chromosome are presented. Quinacrine and trypsin-Giemsa banding identified the extra chromosome as No. 22. The phenotype of these patients and the review of 15 additional similar cases from the literature permit a definition of the cardinal features of trisomy 22: mental and growth retardation, microcephaly and craniofacial asymmetry, strabismus, beaked and prominent nose, long philtrum, cleft palate, micrognathia, large low set ears with preauricular tags and/or pits, long slender fingers, congenital heart disease, inguinal hernia, and hip dislocation.
\end{abstract}

The presence of an extra G-like chromosome in the absence of Down's syndrome has been reported in several instances (for reviews see $\mathrm{Hsu}$ et al, 1971 and Gustavson et al, 1972). Most studies, however, failed to determine the exact identity of the extra chromosome and interpretations of the anomaly included trisomy 21 , trisomy 22, partial D trisomy, partial 18 trisomy, and XYY aneuploidy. Furthermore, the inconsistencies in phenotype among early reported cases added more confusion as to the existence of trisomy 22 as a specific entity. The use of banding techniques in recent studies enabled the diagnosis of trisomy 22 to be made in some cases (Bass et al, 1973; Zackai et al, 1973) but not in others (Gustavson et al, 1972) with similar phenotypes.

In this report we present two patients with an extra $G$ chromosome identified as number 22 by fluorescent and trypsin-Giemsa banding, whose phenotypes strikingly resemble that of some previously reported non-Down's cases with a similar karyotype.

\section{Case reports}

Case 1. This female was born to a 53-year-old father and a 40-year-old mother. A 15 -year-old sister is normal. There were no other pregnancies and the

Received 27 August 1974

* This work was supported in part by grants from the Fundación Endocrinología Infantil and the Consejo Nacional de Investigaciones Científicas y Técnicas. family history was not informative. The gestation of the propositus was complicated by recurrent vaginal bleeding throughout the first trimester, hyperaemesis, and excessive weight gain. Delivery was at term and birth weight was $3200 \mathrm{~g}$. Crying was delayed, she required active measures to stimulate breathing and was placed in an incubator for 12 hours. Her subsequent physical and mental development was poor. She suffered from recurrent gastroenteritis and dehydration and was referred at the age of $\mathbf{4}$ months to our Hospital for evaluation of failure to thrive. At this time her growth was severely retarded (weight $3550 \mathrm{~g}$ ), she was microcephalic (head circumference $37.5 \mathrm{~cm}$ ) and hypotonic. Numerous malformations were apparent on physical examination: cranial asymmetry, with right frontal bossing and left parietal prominence, internal strabismus, long beaked nose, increased philtrum length, cleft hard palate, and micrognathia. The ears were large and low set and there were two preauricular skin tags on the left and a preauricular sinus on the right (Fig. 1). The hands and fingers were long and slender and the thumbs proximally placed (Fig. 2). Bilateral inguinal hernia and left hip dislocation were present. A systolic murmur was heard in the pulmonic area and the ECG showed signs of right ventricular hypertrophy; the presumptive diagnosis of pulmonic stenosis was made.

The patient's cry was hoarse, a direct laryngoscopy showed oedematous vocal cords and thickening of the posterior larynx wall; no abnormalities were found in the trachea. A suprasternal bulbing mass which protruded only in forced expiration (Fig. 1) was interpreted as a mediastinal hernia. The patient was markedly hypotonic and hyporeflexic, could not hold the head still, and had an unexpressive facies. Routine laboratory 

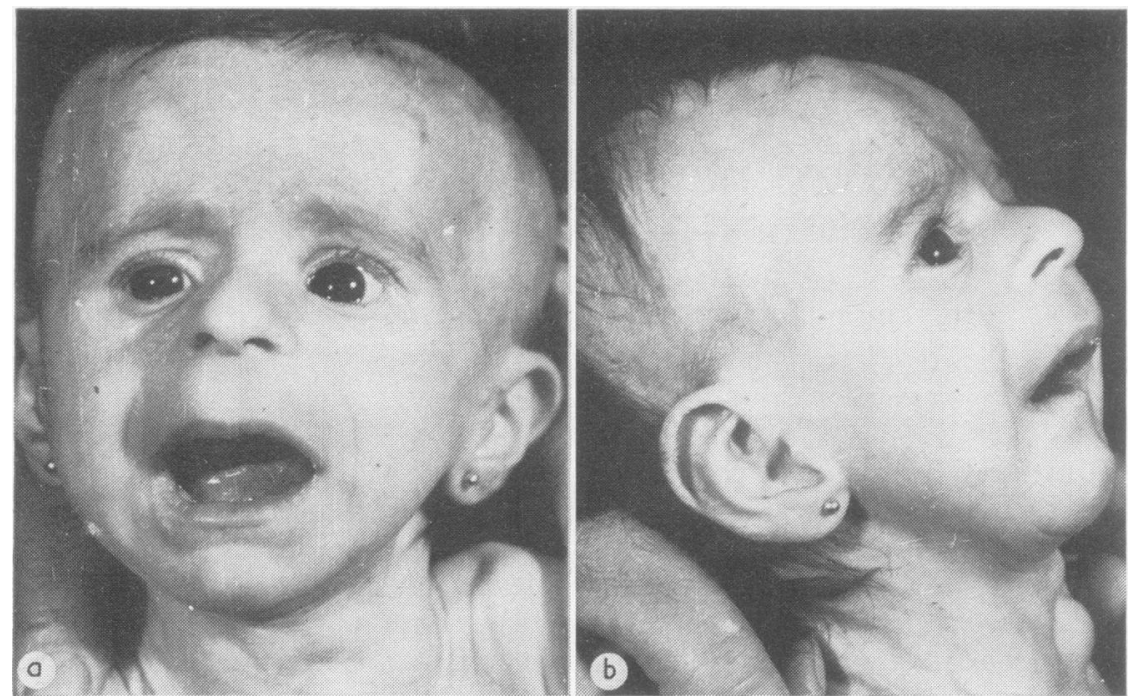

FIG. 1. Case 1 at 4 months of age. Note cranial asymmetry, internal strabismus, long philtrum, left preauricular tags, long beaked nose, long philtrum, micrognathia, preauricular sinus, and protruding suprasternal mass.

analyses including RBC, WBC, blood glucose, blood urea, and urinalysis were all within normal limits. Radiology showed cardiac enlargement, thoraco-lumbar scoliosis, and left hip dislocation.

She was treated for gastroenteritis and dehydration and continued follow-up. Her general health improved although retardation of mental development and physical growth are evident. At the age of 10 months weight was $7100 \mathrm{~g}$ and length was $66.5 \mathrm{~cm}$, she was microcephalic (head circumference $42.8 \mathrm{~cm}$ ) with persistent cranial asymmetry, had marked hypotonia, did not hold the head still nor was she able to sit by herself.

Case 2. This male was the first born of a 26-year-old mother and a 28-year-old father, both normal and non-

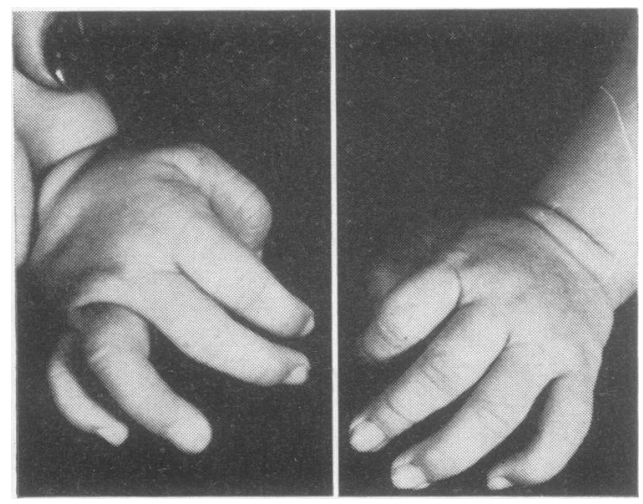

FIG. 2. Hands of case 1 . consanguineous. There were no other pregnancies and the family history was not informative. The propositus was born by Caesarean section at term, weighed $2500 \mathrm{~g}$, and presented delayed crying and cyanosis; a cleft hard palate was detected at birth. Failure to thrive was evident thereafter and cyanosis occurred frequently on crying. Seizures of grand mal type occurred twice during the third month of life. At the age of 5 months a systolic murmur was detected, along with retardation of mental and physical development, generalized hypotonia, and severe malnutrition. He was operated on for a left inguinal hernia at 7 months of age. At 18 months old he was admitted to the Hospital for failure to thrive, hypotonia, and polyuria; the latter had been noted already 2 months before admission. At this time he was severely growth retarded: his length $(74 \mathrm{~cm})$, weight $(6450 \mathrm{~g})$, and head circumference $(43 \mathrm{~cm})$ were all below the 5th centile. He had marked mental retardation and hypotonia, not being able to sit by himself. He performed stereotyped movements of hands and head and his face was unexpressive. Cyanosis was evident in lips and nail beds. Numerous physical abnormalities were evident : microcephaly, facial asymmetry, strabismus, and ptosis, long beaked nose, increased philtrum length, cleft hard palate, micrognathia, large low set ears, preauricular tags bilaterally, and a preauricular sinus on the left (Fig. 3). Hands were thin, fingers long and tapering with increased joint laxity (Fig. 4). Finger and toe nails were hyperconvex and cyanotic. The nipples were low set and a systolic murmur was heard in the pulmonic area. There was a right inguinal hernia and a scar from previous surgery of a left inguinal hernia. He had in addition hypoplastic genitalia, bilateral cryptochidism and left hip dislocation. 

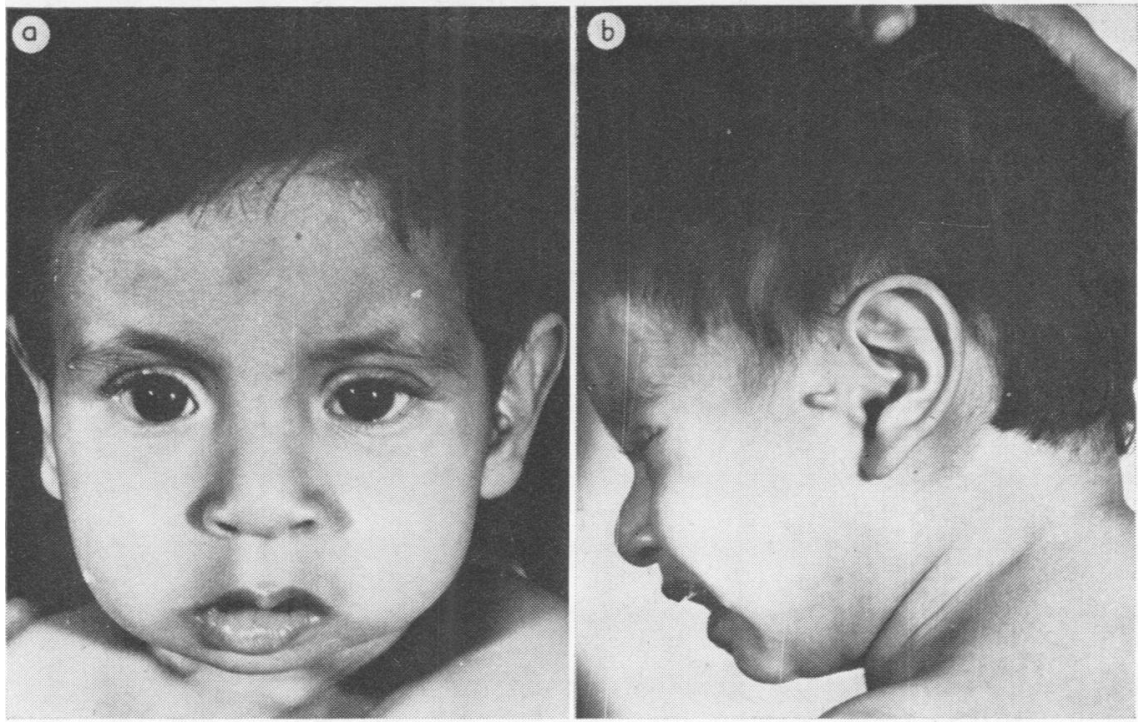

FIg. 3. Case 2 at 18 months of age. Note strabismus, long beaked nose, long philtrum, low set and large ears, and preauricular tags and sinus.

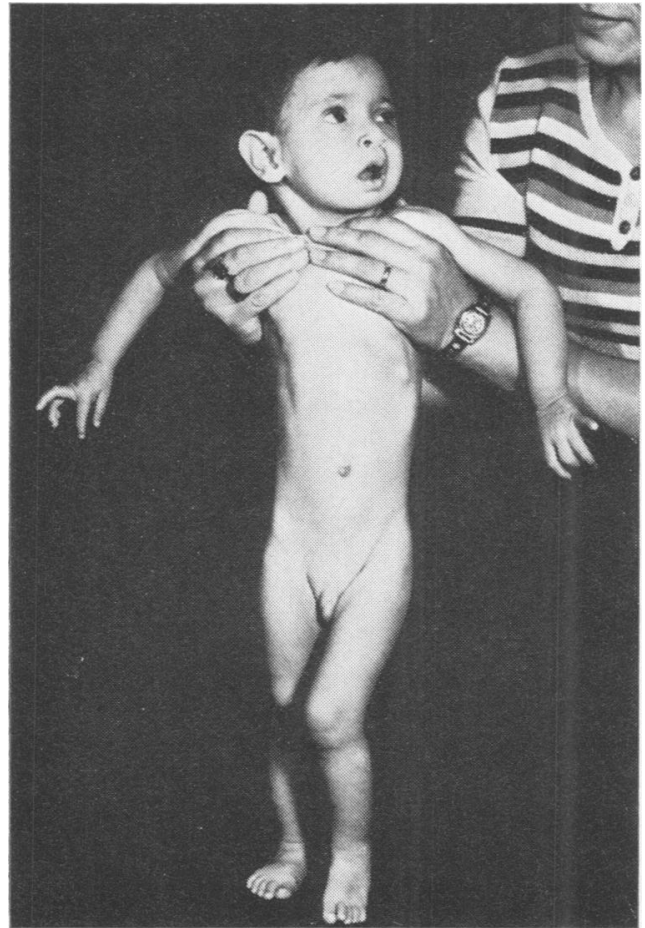

Fig. 4. Case 2. Note general hypotrophy and long slender fingers.
The ECG showed right ventricular load and biventricular hypertrophy. Cardiac catheterization demonstrated a ventricular septal defect and pulmonary stenosis. Initial work-up for his polyuria revealed microhaematuria, mild proteinuria, and high blood urea (10.6 $\mathrm{mmol} / 1$ [64 $\mathrm{mg} \%]$ ). Intravenous pyelogram and renal scan with neohydrine ${ }^{203} \mathrm{Hg}$ failed to show the left kidney and ureter, while the right kidney was apparently normal. Direct bladder vision by cystoscopy demonstrated absence of the left ureteral meatus. The diagnosis of left renal aplasia was thus made. The EEG showed marked diffuse disorganization of slow waves. Radiology revealed microcephaly, cardiac enlargement, and left hip dislocation.

\section{Dermatoglyphics}

The dermatoglyphics of both patients are shown in Table I. The finger patterns of case 2 were hypoplastic.

\section{Cytogenetics}

Chromosome studies in peripheral leucocyte cultures from the two patients and their parents were performed. Both cases had 47 chromosomes and on routine preparations the extra chromosome was undistinguishable from the $G$ group. The chromosome sex constitution was $\mathrm{XX}$ in case 1 and $\mathrm{XY}$ in case 2. No structural abnormalities were detected in the karyotype. The trypsin-Giemsa banding technique (Seabright, 1972) showed two 
TABLE I

DERMATOGLYPHICS

\begin{tabular}{|c|c|c|c|c|c|c|c|c|c|c|}
\hline \multirow{2}{*}{ Case } & \multicolumn{5}{|c|}{ Fingertip Pattern } & \multirow{2}{*}{$\underset{\text { Triradius }}{\text { Axial }}$} & \multirow{2}{*}{$\begin{array}{c}\text { atd } \\
\text { Angle }\end{array}$} & \multirow{2}{*}{$\underset{\text { Line }}{\text { Simian }}$} & \multirow{2}{*}{$\begin{array}{l}\text { Hypothenar } \\
\text { Pattern }\end{array}$} & \multirow{2}{*}{$\begin{array}{l}\text { Thenar } \\
\text { Pattern }\end{array}$} \\
\hline & 1 & 2 & 3 & 4 & 5 & & & & & \\
\hline $\begin{array}{l}\text { Right hand } \\
\frac{1}{2}\end{array}$ & $\stackrel{\mathbb{U}}{\mathbf{W}}$ & $\underset{\mathbf{W}}{\mathrm{UL}}$ & UL & $\underset{\mathbb{W}}{\mathbf{U}}$ & $\begin{array}{l}\text { UL } \\
\text { UL }\end{array}$ & ${ }_{t}^{t, t^{\prime \prime}}$ & $\begin{array}{r}100^{\circ} \\
68^{\circ}\end{array}$ & + & $\overline{-}$ & $=$ \\
\hline $\begin{array}{l}\text { Left hand } \\
1 \\
2\end{array}$ & $\begin{array}{l}\text { UL } \\
\text { UL }\end{array}$ & $\begin{array}{l}\text { UL } \\
\text { UL }\end{array}$ & UL & $\underset{\mathbb{W}}{\mathbf{U}}$ & $\begin{array}{l}\text { UL } \\
\text { UL }\end{array}$ & ${ }_{t}^{t, t^{\prime \prime}}$ & $\begin{array}{l}87^{\circ} \\
65^{\circ}\end{array}$ & + & $\overline{-}$ & $\overline{-}$ \\
\hline
\end{tabular}




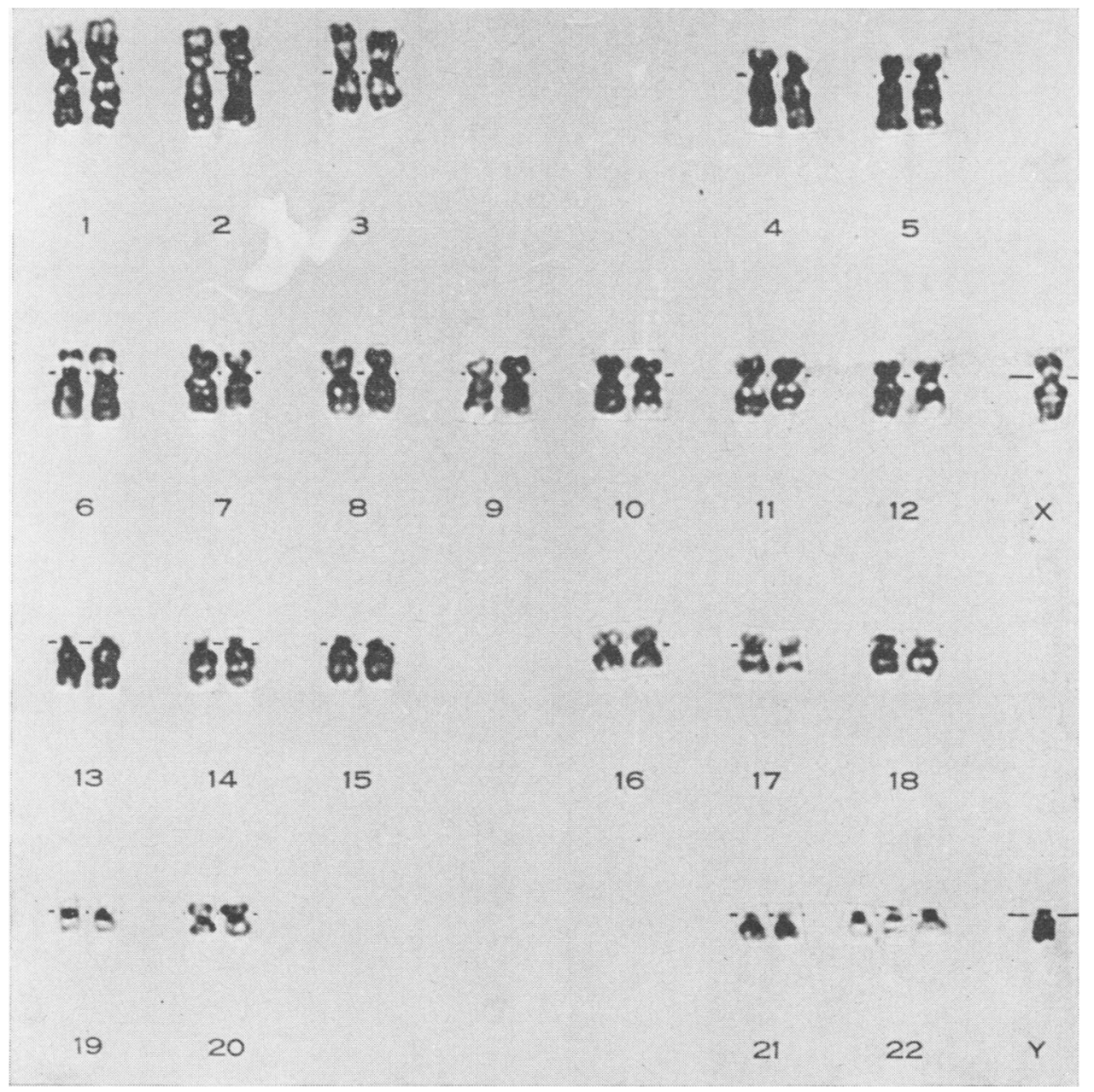

Fig. 6. Chromosomes of case 2 (G banding).

No. 21 chromosomes in the $\mathrm{G}$ group of both cases and three identical No. 22 chromosomes with a narrow band of mild intensity in the middle of long arms. The $\mathrm{Y}$ chromosome of case 2 was clearly distinct from the rest of the G group (Figs. 5, 6, and 7).

The fluorescence banding pattern with quinacrine (Caspersson et al, 1970) also showed two No. 21 chromosomes in both cases and three chromosomes with the characteristics of No. 22 with diffuse fluorescence of mild intensity (Figs. 8 and 9).

The chromosomal analyses of the parents of both patients were normal; no evidence of mosaicism was found.
Discussion

The two cases presented here share a strikingly similar phenotype and an extra G-like chromosome. Quinacrine and trypsin-Giemsa banding techniques permitted identification of the extra chromsome as a No. 22 in both cases. The alternate possibility of a Dq - trisomy appears to have been ruled out on the following grounds: (1) The three chromosomes identified as No. 22 had the same size and were undistinguishable from each other by $Q$ and $G$ banding in both cases. (2) In both patients these three chromosomes had a $\mathrm{G}$ band in the centre of the long arm which was narrower and of lighter staining than that of the proximal segments of the long arms 


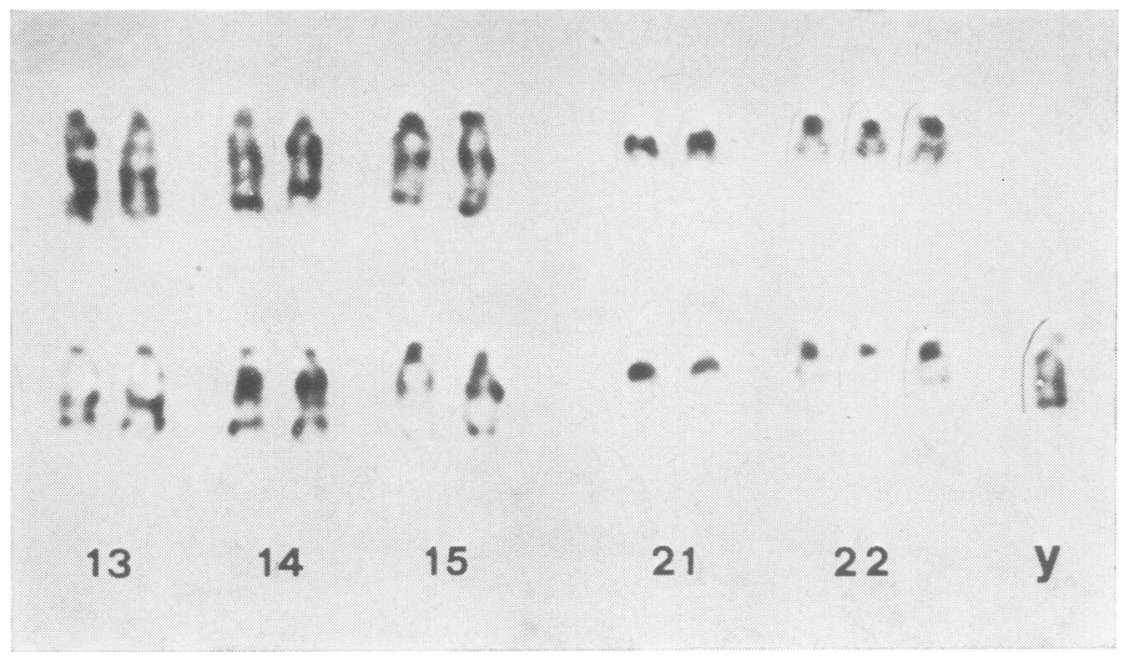

FIG. 7. Partial karyotypes of $D$ and $G$ groups (G banding). Upper row: case 1. Lower row: case 2.

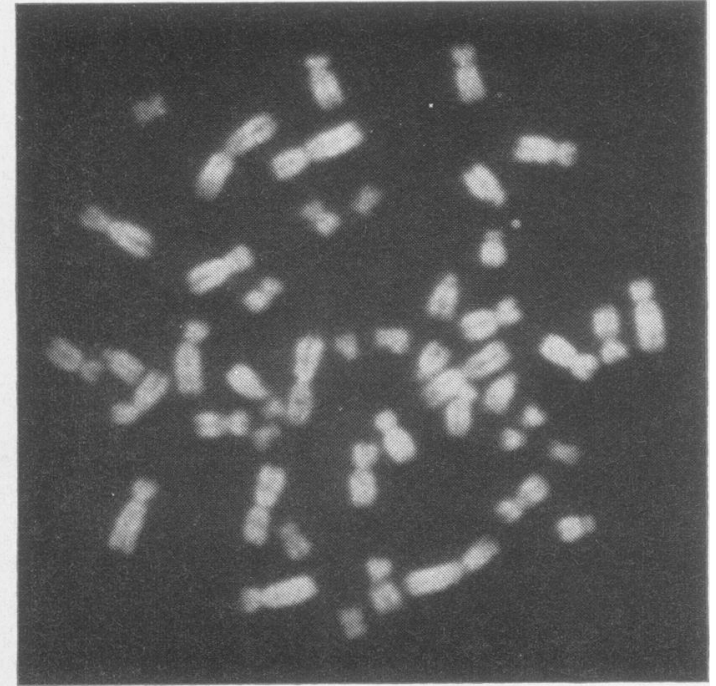

FIG. 8. Metaphase spread of case 1 (quinacrine dihydrochloride stain).

of pairs 13, 14, and 15. (3) Since partial D trisomy is usually the result of unbalanced segregation of a quadrivalent during meiosis, the absence of detectable reciprocal translocation in any of the parents argues against this possibility. (4) A sporadic partial D trisomy would imply the occurrence of two consecutive errors: non-disjunction and deletion. On the other hand, trisomy 22 re-

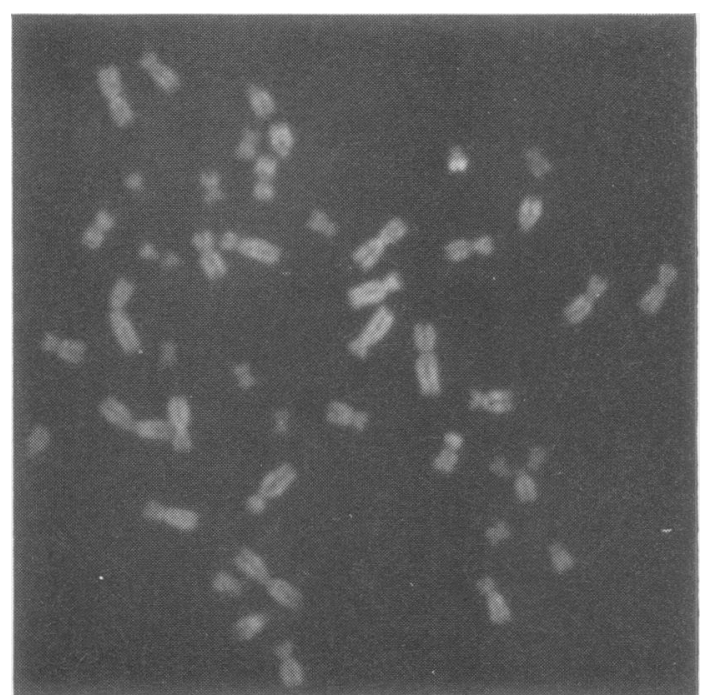

FIG. 9. Metaphase spread of case 2 (quinacrine dihydrochloride stain).

quires for its production only one error: meiotic non-disjunction.

On the basis of these considerations we conclude that our patients have trisomy 22 . The comparison of the present cases with 15 selected non-Down's patients with an extra small acrocentric chromosome reported in the literature (Zellweger et al, 1962; Hall, 1963; Krüger et al, 1968; Uchida et al, 1968; 
TABLE II

MAJOR CLINICAL SIGNS OF TRISOMY 22. BASED ON 17 CASES*

\begin{tabular}{l|c}
\hline \multicolumn{1}{c|}{ Clinical Signs } & Frequencyt \\
\hline Mental retardation & $14 / 14$ \\
Growth retardation & $14 / 14$ \\
Underdeveloped musculature and/or hypotonia & $10 / 12$ \\
Microcephaly & $16 / 16$ \\
Craniofacial asymmetry & $6 / 10$ \\
Long and beaked nose & $7 / 10$ \\
Long philtrum & $11 / 15$ \\
Cleft palate & $14 / 16$ \\
Micrognathia & $13 / 16$ \\
Large low set malformed ears & $14 / 15$ \\
Preauricular tags and/or sinuses & $15 / 17$ \\
Strabismus & $5 / 8$ \\
Long slender fingers and/or finger-like thumbs & $12 / 13$ \\
Congenital heart disease & $12 / 14$ \\
Congenital hip dislocation & $9 / 11$ \\
Cryptorchidism & $5 / 5$ \\
\end{tabular}

* Zellweger et al (1962); Hall (1963); Uchida et al (1968); Krüger et al (1968); Nielsen et al (1969); Hsu et al (1971); Goodman et al (1971); Gustavson et al (1972); Bass et al (1973); Zackai et al (1973), and the present cases.

$t$ When there was uncertainty of the presence of a particular sign, the case was not counted for that sign.

$\ddagger$ The heart malformation is variable. Cardiac catheterization or necropsy in five cases showed different types in each case: persistent ductus arteriosus, atrial septal defect of the secundum type, ventricular septal defect plus persistent ductus arteriosus, ventricular septal lar septal defect plus persistent ductus arteriosus, ventricular septal
defect plus pulmonary stenosis and coarctation of aorta, respectively.

Nielsen et al, 1969; Goodman et al, 1971; Hsu et al, 1971; Gustavson et al, 1972; Bass et al, 1973, and Zackai et al, 1973) reveals a remarkable correspondence in phenotype (Table II). Therefore, and even though some of the authors could come to no definite conclusion about the identity of the extra chromosome, we contend that all those cases are examples of trisomy 22.

The phenotypic spectrum of trisomy 22 (Table II) includes a peculiar aggregation of such stigmata as microcephaly with craniofacial asymmetry, antimongoloid slant of the eyes, ptosis and strabismus, long and beaked nose, long philtrum, malformed ears with preauricular tags and/or sinuses, low set nipples and long slender fingers, along with mental and physical retardation and generalized hypotonia. Additional common findings are cleft palate, micrognathia, heart malformation, inguinal hernia, and congenital hip dislocation. The distinctive features of this entity should probably enable a high degree of diagnostic suspicion on clinical grounds alone. Case 2 of this report had in addition aplasia of the left kidney and renal insufficiency, a finding not reported in previous cases.

It is interesting to note that among the reported cases of trisomy 22 there have been three pairs of sibs with the condition (Uchida et al, 1968; Gustavson et al, 1972, and Zackai et al, 1973), a proportion that seems higher than is usually seen in non-disjunctional chromosome disorders. In some cases this may be the result of parental mosaicism for trisomy 22, as was found in the mother of two sibs with the anomaly (Uchida et al, 1968) and in the mother of a sporadic case (Hsu et al, 1971). In other instances, the presence of atypical parental No. 22 chromosomes could predispose to meiotic nondisjunction; in fact, the mother of the sibs reported by Zackai et al (1973) had a terminal deletion in the long arm of a No. 22. In the rest of the reported cases of trisomy 22 in whom the chromosomes of the parents were studied, including the familial cases of Gustavson et al (1972), no anomalies were found. It will be desirable, however, routinely to screen the parental karyotypes of every further case detected to get more insight into the factors that predispose the production of infants with trisomy 22 .

We are grateful to $\mathrm{Mr}$ Juan Kraizer and Miss Graciela del Rey for skilful technical assistance.

\section{REFERENCES}

Bass, H. N., Crandall, B. F., and Sparkes, R. S. (1973). Probable trisomy 22 identified by fluorescent and trypsin-Giemsa banding. Annales de Génétique, 16, 189-192.

Caspersson, T., Zech, L., and Johansson, C. (1970). Differential binding of alkylating fluorochromes in human chromosomes. Experimental Cell Research, 60, 315-319.

Goodman, R. M., Katznelson, M. B.-M., Spero, R., Shaki, R., Padeli, B., and Sadan, N. (1971). The question of trisomy 22 syndrome. Fournal of Pediatrics, 79, 174-175.

Gustavson, K. H., Hitrec, V., and Santesson, B. (1972). Three non-mongoloid patients of similar phenotype with an extra G-like chromosome. Clinical Genetics, 3, 135-146.

Hall, B. (1963). Mongolism and other abnormalities in a family with trisomy 21-22 tendency. Acta Paediatrica, Suppl. no. 146, pp. 77-91.

Hsu, L., Shapiro, L. R., Gertner, E., Lieber, E., and Hirschhorn, K. (1971). Trisomy 22: a clinical entity. Fournal of Pediatrics, 79, 12-19.

Krüger, E., Wiskowski, R., and Piede, U. (1968). Partielle Trisomie D1-eine seltene Chromosomalomalie. Humangenetik, 6, 181188.

Nielsen, J., Tsuboi, T., Friedrick, U., Mikkelsen, M., Lund, B., and Steinicke, O. (1969). Additional small acrocentric chromosomes: two cases. Fournal of Mental Deficiency Research, 13, 106-122.

Seabright, M. (1972). Human chromosome banding. Lancet, 1, 967.

Uchida, A. I., Ray, M., McRae, K. N., and Besant, D. F. (1968). Familial occurrence of trisomy 22. American fournal of Human Genetics, 20, 107-118.

Zackai, E., Aronson, M., Kohn, G., Moorhead, P., and Mellmam, W. (1973). Familial trisomy 22. (Abstract.) American fournal of Human Genetics, 25, 89-A.

Zellweger, H., Mikamo, K., and Abbo, G. (1962). Two cases of multiple malformations with an autosomal chromosomal aberration-partial trisomy D? Helvetica Paediatrica Acta, 17, 290300 . 